32. This paper was presented at a Symposium on Childhood Cancer at the Annual Meeting of the American Pediatric Society, Denver, Colorado, April 17, 1975.

33. This research was supported in part by Medical Genetics Center Grant no. Center, Houston, Tex. 77030 (USA)
34. Requests for reprints should be addressed to: A. G. Knudson, Jr., M.D., Graduate School of Biomedical Sciences, University of Texas, Health Sciences Center, Houston, Texas 77030 (USA).

35. Accepted for publication July $25,1975$.

\title{
The Role of Cellular Immunity in Neoplasia
}

\author{
PHILIP R. GLADE ${ }^{(94)}$ AND NIEVES M. ZALDIVAR \\ Division of Pediatric Infectious Diseases and Immunology, University of Miami School of Medicine, \\ Miami, Florida, USA
}

LLOYD MAYER AND LINDA T. CAHILL

Mount Sinai School of Medicine, New York, New York, USA

In the past 15 years we have witnessed a rapid evolution in our understanding of the lymphocyte and its role in health and disease. Whereas peripheral lymphocytes had been viewed traditionally as short lived cells with limited biologic activity, it has become abundantly clear that the circulating lymphocyte pool is composed of a spectrum of subpopulations of cells of varying origins, lifespans, fine structural features, and capacities to mediate immunologic responses $(24,25)$. The possibility that immune mechanisms may prevent the development of potentially malignant cells, first formulated by Ehrlich at the turn of the century (18), is one of the most significant outgrowths of this renewed interest in the lymphocyte. The concept of immunologic surveillance, as proposed by Thomas (80) and Burnet (12), suggests that small numbers of tumor cells with altered surface antigens frequently develop in long lived vertebrates, that these cells are recognized as foreign by the cellular immune system of the host, and that they subsequently are eliminated by immunologic mechanisms. Strong supporting evidence for the assumption that neoplastic transformation is accompanied by altered biochemical processes and by the appearance of new surface antigens has been amply provided by our panelists (92). In this segment we will discuss evidence that the tumor-bearing host can recognize and respond to these neoplastic changes. We will consider some aspects of immunosurveillance, the appropriateness of the immune responses detected in the cancer patient, and the potential augmentation of these responses by immunotherapy. In keeping with our charge, these comments will for the most part be limited to a consideration of $\mathrm{T}$ lymphocyte-mediated immune responses.

Clinical evidence that immunologic mechanisms may function in man in the control of cancer comes from multiple sources, most of which indicate a higher incidence of cancer in individuals with depressed or ineffective immunosurveillance. Prior to chemotherapy, patients with malignant disorders frequently manifest a variety of immunologic deficiencies (72). A high incidence of cancer is also noted at the extremes of age when it can be shown that immune systems are less than optimal (12). It has been suggested that in early life the developing and unprimed immune system receives a large battery of antigenic stimulation by "strong" as well as "weak" antigens in the environment. During this time of antigenic bombardment a weakly antigenic malignant cell could escape recognition and establish itself as a tumor. Alternatively, the relatively naive responses of the infant may be more easily overwhelmed by the massive antigenic assault produced by rapidly growing tumor cells. Immunologic responsiveness tends to deteriorate with old age, perhaps (as suggested by Goldstein (23)) weakened by thymic atrophy and decreasing thymosin levels. Of considerable importance is the markedly increased incidence of malignancy in patients with primary immunodeficiency disorders. Neoplasms in these groups occur roughly 10,000 times more frequently than they do in the general age-matched population (21). Cancers affect up to $10 \%$ of patients with certain immunodeficiencies, particularly those involving thymus-dependent $T$ lymphocytes. The development of malignancy is significantly increased in individuals with secondary immunodeficiencies as well.

Patients who have undergone prolonged immunosuppressive therapy for the treatment of disease (including cancer) or to prevent rejection of organ transplants have an incidence of cancer 100 times greater than that of the general population (61). That effective immunosurveillance may be the critical factor in the limitation and final elimination of human cancers is further suggested by the multiple clinical observations of spontaneous regression of established tumors with evidence that tumor immunity has subsequently developed (13). The local accumulation of lymphocytes in certain tumors and, especially, the positive correlation between the intensity of the cellular response and the length of survival provide indirect evidence of in vivo host immune responses to tumors in man (6).

In addition to these clinical observations suggesting lymphocyte participation in the immunosurveillance of human neoplasms is direct evidence of in vivo and in vitro cell-mediated immune responses to a variety of tumor-associated antigens $(31,69)$. Studies have indicated the variable presence of delayed-type hypersensitivity skin reactions to autologous extracts of a variety of human tumors including carcinomas (74), Burkitt's lymphoma (19) and leukemias $(47,60)$. Homologous tumor extracts, however, usually have failed to produce similar skin test reactivity, particularly in patients with Burkitt's lymphoma and leukemia. Reactive 
fractions derived from the carcinoembryonal antigen (CEA) from gastrointestinal adenocarcinoma have produced positive skin test responses in allogeneic as well as autologous patients with intestinal cancer (34). Fractionation studies have shown that this skin-reactive antigen and CEA are distinct (35). Black and his colleagues $(4,5)$, using skin window techniques, have further demonstrated that patients with breast cancer will respond to autologous breast cancer tissue, but not to normal breast tissue.

The existence of cell-mediated immunologic reactions against antigens associated with human neoplasms has also been demonstrated by numerous in vitro assays. With cytotoxicity techniques cell-medaited immune responses against autologous tumor antigens have been observed in patients with carcinoma of the colon (29), nasopharynx (14), urinary bladder (11), breast (30), lung (30), kidney (17), testes (30), and endometrium and ovary (30). Similar immune cytotoxic reactivity has been described for patients with malignant melanoma (30), with lymphomas (67), and with sarcomas $(30,67)$. Direct cytotoxic reactivity against fresh or frozen viable leukemic cells has been demonstarated as well $(33,47)$. Lymphocyte transformation studies have been useful in demonstrating cell-mediated immune responses in some individuals with Burkitt's lymphoma (77), sarcomas (82) and carcinomas of the kidney (75), lung (78), and testes (76). There is no apparent correlation, however, between positive lymphocyte stimulation and the clinical state of the patient $(75,82)$. Direct leukocyte migration inhibition by extracts of tumor has also been reported in patients with breast cancer (2) and other carcinomas $(2,64,89)$.

Supporting in vitro evidence that the tumor-bearing host can recognize neoplastic changes and can respond in immunologic ways continues to proliferate at a prodigious rate $(13,32,38,69)$. Outgrowth of studies with the lymphocyte transformation model has furthermore provided us with plausible mechanisms whereby we can envisage how this recognition and activation of lymphocytes might culminate in tumor destruction. A central problem to an understanding of cell-mediated immunity has been the elucidation of the effector mechanisms by which specifically sensitized lymphocytes, in their response to antigens, recruit inflammatory cells and induce tissue damage. Definitive studies, not possible with. the insufficient materials obtained from in vivo skin test sites, required first the development of adequate in vitro methods which mimic the responses associated with deiayed-type hypersensitivity in vivo and correlate with the cutaneous reactivity of the donor. The similarity of phytohemagglutin (PHA)-transformed lymphocytes in short term culture to the large pyroninophilic cells of the paracortical regions of lymph nodes draining areas of antigenic challenge in animals sensitized for cell-mediated immunity quickly captured the interest of cellular immunologists. In time it became quite clear that the PHA responsiveness of lymphocytes in short term culture generally reflects the integrity and competence of the cellular immune system of the host (59). Of particular interest, it was similarly shown that lymphocytes from sensitized donors undergo activation and blastogenic responses when cocultured with a variety of antigens and that this antigenic stimulation of lymphocytes in vitro closely correlates with the in vivo skin test reactivity of the individual (62). Intensive research efforts in many laboratories in the past few years have subsequently led to the discovery that this activation of small lymphocytes in vitro is attended by the release of a variety of potent, soluble materials, including a migration inhibitory factor $(10,15)$; a skin reactive factor $(8,63)$, lymphotoxin (39), a mitogenic factor (37), chemotactic factors for neutrophils (86) and mononuclear cells (85) and interferon (88). These factors have the in vitro capacity to recruit nonsensitized cells and to cause cell damage in ways that rather closely reflect the various responses associated with cell-mediated immunity in vivo. It is postulated that these are the soluble mediators which orchestrate in vivo the sequence of events leading to induration and augmented responses to antigenic differences on a cellular level (44) and resulting in the localization and destruction of cells with altered surface antigenicity (52).

Despite strong supporting evidences in man for the fundamental assumptions of the immunosurveillance theory and the definition of potential mechanisms for the destruction of tumor cells by activated lymphocytes, actual progress in our understanding of the human malignant process and in the development of the immunologic means for its control and cure have been agonizingly slow and inept. How often immunosurveillance mechanisms prevent carcinogenesis is unknown. That they frequently fail is obvious (41). Particularly distressing is that they often fail despite the existence of a precancerous phase of some tumors for years, e.g., solar keratoses and leukoplakia preceding invasive squamous carcinoma; lentigo maligna preceding malignant melanoma; intestinal polyps preceding adenocarcinoma of the colon; dysplastic squamous epithelial lesions of the cervix and of the bronchial tree preceding carcinomas of the cervix and of the lung; atypical endometrial hyperplasia preceding endometrial carcinoma; and hydatidiform mole leading to choriocarcinoma. Immunosurveillance for these precancerous lesions and for their resulting malignant processes seems to be totally absent or ineffective, despite a prolonged latency period. Equally distressing for proponents of the immunosurveillance theory is the development of neoplasms in sites of chronic inflammation where large numbers of competent and activated cells abound, e.g., adenocarcinoma of bowel arising in patients with ulcerative colitis, squamous carcinoma arising in burn scars and in bladder schistosomiasis, carcinomas and sarcomas in osteomyelitis, and carcinoma of stomach in atrophic gastritis. Similarly confusing is the metastatic implantation of tumor is draining lymph nodes, usually in the cortical sinusoids where $T$ lymphocytes are most numerous.

Multiple immunologic mechanisms have been implicated in these failures of immunosurveillance. It is possible, as has been demonstrated for the tumors induced by chemical carcinogens, that some human cancers are devoid of tumor-associated antigens. Immunosurveillance mechanisms may actually select for these aberrant clones (7). As demonstrated by neuraminidase treatment, some tumor antigens may be submerged in the cellular membrane or "hidden" within the glycoprotein complex (87). Some tumors may have such weak antigens that they are missed unless the immune system is alerted by the coincident presence of additional nonrelated antigens which gear up the response. Conceivably, this "sneaking through" may also be promoted by oncogenic agents that preoccupy the host immunologically or that stimulate the target tissue harboring the neoplastic clone to an inordinately rapid proliferation, e.g., ulcerative colitis and adenocarcinoma of colon; malaria and Burkitt's lymphoma. A large body of evidence has recently accumulated which demonstrates the blocking by serum factors of otherwise cytotoxic lymphocyte responses (28). It was originally assumed that this phenomenon resulted from blocking antibodies which coated tumor surfaces and shielded them from $T$ cell surveillance. It now appears that blocking factors are antigens and antigen-antibody complexes which effectively block the $\mathrm{T}$ cell receptor site (68). Failure of immunosurveillance in the gross immunodeficiencies, particularly the $\mathrm{T}$ cell disorders, results in a high incidence of cancer in these groups. The rarity of these conditions makes this an unlikely mechanism for many cancers in the general population. Cancer could result, however, from highly selective defects in immunologic surveillance (40). These selective defects could be the consequence of genetically determined unresponsiveness, perhaps linked to histocompatibility genes akin to the Ir genes in mice (54). Selective defects could be induced by the establishment of a state of low threshold tolerance as well, reflecting faulty T-B cell interactions. Disordered T-B cell interaction may also be at fault in the lymphoreticular malignancies (22)

Central to any pragmatic understanding of the role of cellmediated immunity in human neoplasia is the detection and characterization of "tumor-specific" antigens and, in turn, the definition of the immune response induced by them which are protective for the host. Although such antigens have been described for small mammals, tumor-specific antigens have yet to be defined for human neoplasms. Investigations of effective tumor 
immune responses in man are further hampered by our persistence in looking for protective mechanisms in patients with established noeplasms. If cell-mediated immune responses are the basis for the rapid detection and elimination of malignant cells from the body, then the presence of cancer in an individual must be viewed as his demonstrated failure to muster appropriate immune responses. The immune reactivities to tumor-associated antigens in the cancer-bearing patient may have little to do with effective immunosurveillance. The critical issue may be what is lacking in these individuals. Perhaps protective tumor immunities should be sought in healthy populations at risk for a malignant process, where there may be individuals who have successfully rejected the neoplasm in its early stages (41). It is reasonable as well as to suspect that chronic inflammatory disorders may represent the nonneoplastic cellular attempts to deal with an otherwise carcinogenic stimulus or insult in the immunocompetent individual who can muster specific, overly aggressive, and enduring immune reaction. Such individuals may offer the most useful populations to search for the missing immune reactivities in patients with malignancies of similar tissues.

Of the chronic inflammatory bowel disorders, ulcerative colitis and granulomatous colitis may be viewed as polar aspects of the spectrum of gastrointestinal diseases leading to adenocarcinoma of colon. The cancer risk in all cases of ulcerative colitis is 9 times that of the general population, with an overall incidence of $3-5 \%$. The three highest risk factors are extensive colonic involvement, a disease duration of 10 or more years, and onset before 18 years of age (51). An inflammatory colitis similar to Crohn's disease of the small intestine has also been described. This entity, referred to as Crohn's disease of the large bowel or granulomatous colitis, is characterized clinically by fever and abdominal pain with a course marred by anorectal complications highlighted by fistulas and abscess formation. Unlike ulcerative colitis, which is mainly a mucosal inflammation, Crohn's disease initially affects the submucosa, with gradual transmural extension. The development of carcinoma in areas involved by Crohn's disease is felt to be uncommon, although there have been studies revealing the rare coexistence of cancer and Crohn's disease (20). It is felt that immunologic mechanisms play a major role in the pathogenesis of both ulcerative colitis and granulomatous colitis. We, as others (65), have detected the presence of tumor-associated CEA in the peripheral circulation of patients with ulcerative colitis and granulomatous colitis. It is presumed that in these chronic inflammatory diseases of intestines the increased turnover rate of mucosal cells associated with inflammation results in the appearance of cells with increased surface antigenicity (CEA). It seemed reasonable to postulate that the development of digestive system tumors in these patients should depend in part upon the efficiency of host immune responses to recognize these changes in surface antigenicity associated with adenocarcinoma and to respond to cells which bear them. It is reasonable to expect, therefore, that immune responses should distinguish low cancer incidence groups with Crohn's disease from high incidence groups with ulcerative colitis and patients with bowel cancer.

In an attempt to define some of the differences in immunologic capacity of patients within this spectrum of diseases affecting the gastrointestinal tract, we assessed the in vitro inhibition of migration of peripheral leukocytes from these patients by a CEAcontaining "antigen" derived from human meconium. Thirty-four normal individuals, 21 patients with adenocarcinoma of the colon, 23 patients with ulcerative colitis, and 39 patients with granulomatous colitis were investigated. All patients with adenocarcinoma of the colon had tissue diagnoses. Patients were diagnosed as having nonspecific ulcerative colitis and granulomatous colitis according to generally accepted clinical and morphologic criteria. No patient had significant hepatic or renal disease and there was no evidence of malignancy in the group with inflammatory disease. None of the patients was receiving corticosteroids or antimetabolite medications.

Table 1 summarizes the results of the inhibition of migration of fresh peripheral leukocytes from our study patients in the presence of meconium. Migration inhibition of less than 20\% was not considered significant. Normal subjects showed no significant inhibition of leukocyte migration with the CEA-containing meconium antigen. Few of the cancer patients had inhibitions of migration of their leukocytes in the presence of meconium antigen $(14 \%)$. In contrast, most patients with Crohn's disease $(93 \%)$ demonstrated inhibition of leukocyte migration in the presence of this material. In the ulcerative colitis group, approximately a third (31\%) showed similar leukocyte migration inhibition. These studies with leukocytes from normal individuals and from patients with Crohn's disease, ulcerative colitis, and carcinoma of the colon suggest that differences exist in the immune responses of these groups of patients to human meconium containing a CEA-like substance. Leukocytes from most normal individuals (presumably unchallenged as yet in adult life) and from most patients with carcinoma of the bowel (perhaps tolerant or genetically unresponsive) fail to recognize some component of this material and their migration is unaffected by it. Leukocytes from patients with Crohn's disease, however, are uniformly inhibited. Since the incidence of carcinoma of the bowel in patients with Crohn's disease is quite small, this reactivity is at least potentially related to the demonstrated effective immunosurveillance for adenocarcinoma of the bowel in these patients. It is equally possible that this response could lead to progressive tissue damage and the chronic inflammatory condition which perpetuates Crohn's disease. Leukocytes from two-thirds of the patients with ulcerative colitis, a disease with a high cancer risk, behave similarly to those of adenocarcinoma patients and fail to recognize this meconiumderived antigen. If the response to this material is potentially protective, as data from granulomatous colitis patients might suggest, then we can speculate that the lack of such response might also be predictive of those individuals with ulcerative colitis at risk to develop adenocarcinoma of bowel.

The exact nature of the antigen which elicits the differential responseiveness in the leukocytes of these patients remains to be determined. Studies with purified CEA unfortunately show no inhibition of leukocyte migration in these patients (79). It is known, however, that delayed-type hypersensitivity responses are hapten-carrier specific, whereas serologic responses are critical only for the hapten moiety (66). Leukocytes from purified protein derivative (PPD)-sensitive individuals show marked migration inhibition in the presence of intact mycobateria (71), but no consistent patterns are found for soluble PPD (50). It is possible that the extraction and purification procedures used to isolate CEA alter specificity for cell-mediated responses without affecting serologic reactivity. It is also possible that CEA represents a family of closely related antigens of varying immunologic specificity and that different disorders are associated with specific antigens within the CEA family. Further investigation of the nature of the meconium antigen employed in this leukocyte migration inhibitory test is clearly indicated. It is quite possible that it not only will differentiate a wide spectrum of gastrointestinal disorders, but also may be predictive for individuals particularly at risk to develop adenocarcinoma. Studies in the aggressively immunoreative patient may provide the investigative tools to detect responses which are defective or missing in the cancer patient and, perhaps, the appropriate transfer factors for effective immunotherapy for malignant disease.

Table 1. Inhibition of leukocyte migration by meconium "antigen"

\begin{tabular}{lcc}
\multicolumn{1}{c}{ Subjects } & Migration inhibition & \multirow{2}{*}{$\%^{\prime}$} \\
\hline Adenocarcinoma of bowel & $(3 / 21)$ & 14 \\
Ulcerative colitis & $(7 / 23)$ & 31 \\
Granulomatous colitis & $(36 / 39)$ & 93 \\
Normal & $(1 / 34)$ & 3
\end{tabular}

${ }^{1}$ Inhibition of $20 \%$ or more of control migrations. 
Armed with accumulating data demonstrating the presence of cell-mediated immune responses against tumor-associated antigens in man, the possibility of altering the course of cancer by manipulation of host defense mechanisms has become increasingly attractive to immunologists and to physicians charged with the care of these patients. The possibilities and problems of immunologic intervention in cancer are manifold, as elaborated in a review by Smith (70). Four avenues for augmentation of tumor immunity were espoused, including: (I) nonspecifically augmenting cellular immunity; (2) increasing the effectiveness of specific cell-mediated immunity; (3) interfering with blocking serum factors; (4) increasing macrophage interaction and specific antibodies. Of these postulated modes of intervention, the increments of nonspecific and of specific cell-mediated immune reactivity of the cancer-bearing patient against his neoplasm offer the most promising approaches.

After the demonstration that bacillus Calmette-Guérin (BCG) vaccine is a potent nonspecific immunostimulant in a variety of animal tumor models (58) and the epidermologic evidence of decreased incidence of lymphomas and leukemias in countries with routine BCG immunization programs (16), clinical trials of this form of nonspecific adjuvant immunotherapy have been conducted in patients with leukemia (53) and with melanoma (56). The initial clinical results were encouraging (3), but were empirical in regard to dose, route, schedule, and strain of BCG preparation used. Recent studies suggest that high dose scarification with Tice strain BCG may offer better therapeutic responses (27). Best results occurred when the BCG was injected directly into the malignancy (56). This nonspecific augmentation of cell-mediated immune responses is far from efficient, however, and BCG is associated with significant risks $(26,73)$. It frequently causes inflammation and abscess formation at the infection sites. Recurrent fevers with generalized symptoms of malaise and gastrointestinal distress are common. Liver abnormalities and, on occasion, severe hypersensitivity reaction can occur.

Because of BCG's variable efficacy and demonstrated risk, investigators have begun to explore the use of other nonspecific adjuvants. These include membrane fragments and extracts of BCG (55), Corynebacterium parvum (36), and levamisole (81). C. parvum, effective in preventing tumors in animals, has practical advantages over BCG. It is readily standardized, nonviable, and generally well tolerated in man in effective doses. $C$. parvum has been shown to stimulate production of large numbers of macrophages which seem to mediate most of its antitumor effects. Its efficacy for the adjuvant immunotherapy of human tumors is as yet essentially unknown. Levamisole, an effective anthelminthic with few toxic effects, seems capable of nonspecifically stimulating immune responses as well. Although the mechanism is unknown, it is probable that macrophages are involved in some of the actions of levamisole. Clinical usefulness for levamisole has been claimed in a wide range of disorders (46). Several studies of levamisole in the adjuvant immunotherapy of melanoma (83) and bronchiogenic carcinoma (1) are in progress and suggest that this form of therapy may be useful.

An additional class of "nonspecific" adjuvants considered for potential cancer immunotherapy includes thymosin and transfer factor. Both substances have been shown to be clinically useful in the partial reconstitution of nonspecific cellular immunity in patients with immunodeficiencies $(9,84)$. Each appears to have the capacity to increase the percentages of $T$ cell rosettes in the peripheral circulation $(84,90)$. Recent observations in our laboratory further suggest that transfer factor many be useful in some forms of agammaglobulinemia by facilitation of $T-B$ cell lymphocyte interactions (91). This nonspecific effect of transfer factor is presently under investigation by us in the therapy of the $B$ cell dyscrasias (Waldenström's macroglobulinemia and multiple myeloma).

A reasonable alternative mode of immunotherapy with potential to augment specific immune reactivities in the recipient is transfer factor. Lawrence and coworkers $(42,43)$ have amply demonstrated that an extract of circulating human lymphocytes has the capacity to convert nonsensitized recipients to an antigen-responsive state in vivo. This material, transfer factor, confers on the nonsensitive human recipient and his lymphocytes the pattern of delayed skin reactivity of the donor and, therefore, the specific cellular immunologic memories the donor possesses. The delayed sensitivity transferred is characterized by prompt onset (hours to days), prolonged duration (months to 1 or 2 years), and systemic distribution. Transfer factor is a soluble, dialyzable, lyophilizable material of less than 10,000 molecular weight. It contains no albumin or $\gamma$-globulin, is orcinol-positive, and is of a polypeptidepolynucleotide composition. It is dissociable from transplantation antigens and it is not immunogenic. Parenteral use of transfer factor is associated with few side effects and has therapeutic value in a variety of disease states with defective cell-mediated immunity, including disseminated vaccinia, chronic mucocutaneous candidiasis, lepromatous leprosy, Wiskott-Aldrich syndrome, and common variable immunodeficiency (43).

Growing evidence demonstrates that reconstitution with transfer factor of general cell-mediated immunologic functions in patients with a variety of neoplastic diseases is possible (42). Most $(85 \%)$ anergic patients with acute leukemia, chronic leukemia, lymphosarcoma, and carcinomas studied are capable of responding to transfer factor with the development of delayed cutaneous reactivity to tuberculin or to streptokinase-streptodornase. These studies suggest that the cellular capacity to respond to transfer factors remains intact in many groups of immunodepressed cancer patients and that transfer factors are potentially of use in the specific immunotherapy of these conditions. A few therapeutic trials with dialyzable transfer factor in selected patients with breast cancer, melanoma, and osteogenic sarcoma have been attempted in recent years $(42,48,49,57)$. Most of these are incomplete and yield only suggestive data. One of five patients with breast cancer was felt to have some clinical response to pooled nonspecific transfer factor from normal, healthy females. Therapy with specific transfer factors from long term melanoma survivors appear to offer more beneficial effects with documented regression and rejection of metastatic melanoma nodules. The vagaries of the natural course of melanoma, however, make firm statements of the "effects" of transfer factor in this disease difficult. Continuing studies with transfer factors from long term survivors of breast cancer suggest that these preparations may inhibit the growth of advanced breast cancer and may be of therapeutic use in patients with minimal residual breast cancer (49). We have similar findings in patients treated at Mount Sinai School of Medicine.

Studies of the mechanism of homograph rejection in man have clearly underlined the necessity of employing a specific transfer factor to cause rejection of the appropriate target graft (45). This specificity is likely to apply to tumor rejection as well. The effectiveness of therapy will undoubtedly depend upon the immunologic specificity of the transfer factor employed, the dosage used, and the duration of therapy required to re-arm the lymphoid population of the host, or, perhaps, to break his tolerance to the tumor. The selection of appropriate transfer factors, i.e., appropriate cell-mediated immune responses against tumor or its products, therefore, is of critical importance in any further clinical trials of this mode of immunotherapy. It is quite possible that the appropriate donors of these specific transfer factors may be individuals with chronic inflammatory disorders rather than the long term survivors of neoplastic disease.

There is at hand ample and compelling clinical and laboratory evidence documenting the existence of a cell-mediated immunosurveillance mechanism in man which plays a central role in the pathogenesis and course of human cancers. Although the immunologic mechanisms which contribute to the failure of immunosurveillance in overt cancer are still largely undefined, we are developing efficient ways to effect immune responses in the tumor-bearing patient. It is clear that we must now begin to sort out specific and protective immune responses in the cancer patient and in the general population which deserve restoration or 
augmentation. With this knowledge should come the long anticipated era of cancer immunotherapy and preventitive immunization.

\section{REFERENCES AND NOTES}

1. Amery, W.: Levamisole. Lancet, $i: 389$ (1975)

2. Andersen, V., Bjerrum, O. Bendexen, G., Schiodt, T., and Dissing, 1.: Effect of autologous mammary tumor extracts on human leukocyte migration in vitro. Int. J. Cancer, 5: 357 (1970).

3. Bast, R. C., Zbar, B., Borsos, T., and Rapp, H. J.: BCG and cancer. N. Engl. J. Med., 290: 1413, 1458 (1974).

4. Black, M. M.: Human breast cancer: A model for cancer immunology. In: D. W Weiss: Immunological Parameters of Host Tumor Relationships, Vol. 2, p. 80 Academic Press, New York (1973)

5. Black, M. M., and Leis, H. P.: Cellular responses to autologous breast cancer tissue: Correlation with stage and lymphoreticuloendothelial reactivity. Cancer 28: 263 (1971)

6. Black, M. M., Opler, S. R., and Speer, F. D.: Microscopic structure of gastric carcinomas and their regional lymph nodes in relation to survival. Surg. Gynecol Obstet. 98: 725 (1954)

7. Black, M. M. Zachman, R. E., Shore, B., Moore, D. H., and Leis, H. P. Prognostically favorable immunogens of human breast cancer tissue Antigenic similarity to murine mammary tumor virus. Cancer, 39: 121 (1975).

8. Bennett, B., and Bloom, B. R.: Reactions in vivo and in vitro produced by a soluble substance associated with delayed-type hypersensitivity. Proc. Natl. Acad. Sci. U.S.A., 59: 756 (1968).

9. Bloom, B. R.: Does transfer factor act specifically or as an immunologic adjuvant? N. Engl. J. Med., 288: 908, (1973).

10. Bloom, B. R., and Bennett, B.: Mechanisms of a reaction in vitro associated with delayed-type hypersensitivity. Science, 153: 80, (1966)

11. Bubenik, J., Perlmann, P., Helmstein, K., and Moberger, G.: Cellular and humoral immune responses to human urinary bladder carcinomas. Int. J. Cancer, 5: $310(1970)$

12. Burnet, F. M.: Immunological recognition of self. Science, 133: 307 (1961).

13. Burnet, F. M.: Immunological Surveillance. (Pergamon Press, Oxford (1970).

14. Chu, E. H. Y., Stjernswärd, J., Clifford, P., and Klein, G.: Reactivity of human lymphocytes against autochthonous and allogeneic normal and tumor cells in vitro. J. Nati. Cancer Inst., 39: 595 (1967)

15. David, J. R.: Delayed hypersensitivity in vitro: Its mediation by cell-free substances found by lymphoid cell-antigen interaction. Proc. Natl. Acad. Sci. U.S.A., 56: 72 (1966)

16. Davignon, L., Lemonde, P., Robillar, P., and Frappier, A.: B. C. G. vaccination and leukemia mortality. Lancet ii: 638 (1970).

17. Diehl, V., Jereb, B., Stjernswärd, J., O'Toole, C., and Ahström, C.: Cellular immunity to nephroblastoma. Int. J. Cancer, 7: 277 (1971)

18. Ehrlich, P.L In: Ober den jetzigen stand der Karzinom forschung. Nederlandsch Tijdschift voor Genuskunde. Erste Helft, No. 5 (1909).

19. Fass, L., Herberman, R. B., and Ziegler, J.: Delayed cutaneous hypersensitivity reactions to autologous extracts of Burkitt lymphoma cells. N. Engl. J. Med. 282: $776(1970)$

20. Frank, J. D., and Shorey, B. A.: Adenocarcinoma of the small bowel as a complication of Crohn's disease. Gut, 14: 120 (1973).

21. Gatti, R. A., and Good, R. A.: Occurrence of malignancy in immunodificiency diseases. Cancer 28:89 (1971)

22. Gershwin, M. E., and Steinberg, A. D.: Loss of suppressor function as a cause of lymphoid malignancy. Lancet ii: 1174 (1973).

23. Goldstein, A. L., Slater, F. D., and White, A.: Preparation, assay, and partial purification of a thymic lymphocytopoietic factor (thymosin). Proc. Natl. Acad. Sci. (U. S. A., 56: 1010 1966)

24. Good, R. A.: Immunodeficiency in developmental perspective. Harvey Lect., 67: 1 (1973).

25. Gowans, J. M., and McGregor, D. D.: the immunological activities of lymphocytes. Progr. Allergy, 9: 1 (1965).

26. Grant, R. M., Mackie, R., Cochran, A. J., Murray, E. L., Hoyle, D., and Ross, C.: Results of administering B.C.G. to patients with melanoma. Lancet, $i i: 1096$ (1974).

27. Gutterman, J., Mavligit, G., McBride, C., Frei, E., III, and Hersh, E. M. B.C.G. stimulation of immune responsiveness in patients with malignant melanoma. Cancer 32: 321 (1973)

28. Hellström, K. E., and Hellström, I.: Lymphocyte mediated cytotoxiciity and blocking serum activity to tumor antigens. Adv. Immunol., 18: 209 (1974).

29. Hellström, I., Hellström, K. E., and Shepard, T. H.: Cell-mediated immunity against antigens common to human colonic carcinomas and fetal gut epithelium. Int. J. Cancer, 6: 346 (1970)

30. Hellström, I., Hellström, K. E., Sjögren, H. O., and Warner, G. A.: Demonstraion of cell-mediated immunity to human neoplasms of various histological types. Int. J. Cancer, 7: 1 (1971)

31. Herberman, R. B.: Cellular immunity to human tumor-associated antigens Israel J. Med. Sci., 9: 300 (1973)

32. Herberman, R. (Ed): Conference and Workshop on Cellular Immune Reactions to Human Tumor-Associated Antigens. Natl. Cancer Inst. Mongr., 37 (1973).

33. Herberman, R. B., and Rosenberg, E. B.: Cellular cytotoxicity reactions to human leukemia associated antigens. In: Proceedings of the Fifth International Symposium on Comparative Leukemia Research, 1972.
34. Hollinshead, A. C., and Herberman, R. F.: Separation of major histocompatibility antigens from other antigens present on human leukemic and white blood cell membranes. In: Proceedings of the Fifth International Symposium on Comparative Leukemia Research, 1972.

35. Hollinshead, A., McWright, C., Alford, I. C., Glew, D., Gold, P., and Herberman, R.: Separation of skin reactive intestinal cancer antigen from the carcinoembryonic antigen of Gold. Science 177: 887 (1972).

36. Immunological control of cancer. Lancet, i: 502 (1975).

37. Kasakura, S., and Lowenstein, L.: A factor stimulating DNA synthesis derived from the medium of leukocyte cultures. Nature, 208: 794 (1965).

38. Klein, G.: Tumor-specific transplantation antigens. G. H. A. Clowes Memorial lecture. Cancer Res., 28: 625 (1968).

39. Kolb, W. P., nd Granger, G. A.: Lymphocyte in vitro cytotoxicity: characterization of human lymphotoxin. Proc. Natl. Acad. Sci. U. S. A., 61: 1250 (1968).

40. Laroye, G. J.: Cancer caused by an inherited selective defect in immunological surveillance. Lancet, $i$ : 641 (1973).

41. Laroye, G. J.: How efficient is immunological surveillance against cancer and why does it fail? Lancet $i: 1097$ (1974).

42. Lawrence, H. G.: In: C. H. Kirkpatrick and D. Rifkind: Workshop on Basic Properties and Clinical Applications of Transfer Factor. Department of Health, Education and Welfare, United States Public Health Service, National Institutes of Health, 1974.

43. Lawrence, H. S.: Transfer factor in cellular immunity. Harvey Soc., 68: 239 (1972-73)

44. Lawrence, H. S., and Landy, M. (Eds.): Mediators of Cellular Immunity (Academic Press, New York, 1969).

45. Lawrence, H. S., Rapaport, F. T., Converse, J. M., and Tillett, W. S.: Transfer of delayed hypersensitivity to skin homografts with leukocyte extracts in man. J. Clin. Invest., 39: 185 (1960)

46. Levamisole. Lancet, $i: 151$ (1975).

47. Levanthal, B. G.: Halterman, R., Rosenberg, E. G., and Herberman, R. B.: In vitro and in vivo immunological reactivity against autochthonous leukemia cells. Cancer Res., 32: 1820 (1972)

48. Levin, A. S., Buyers, V. S., Fudenberg, H. H., Wybran, J., Hackett, A. J., Johnston, J. O., and Spitler, L. E.: Osteogenic sarcoma: Immunologic parameters before and during immunotherapy with tumor specific transfer factor. J Clin. Invest. 55: 487 (1975)

49. LoBuglio, A. F., and Neidhart, J. A.: A review of transfer factor immunotherapy in cancer. Cancer, 34: 1563 (1974).

50. Lockshin, M. D.: Failure to demonstrate leukocyte inhibition in human tuberculin hypersensitivity. Proc. Soc. Exp. Biol. Med. 132: 928 (1969).

51. Loskhart-Mummery, H. E.: Diffuse conditions of the large bowel which are premalignant. Brit. J. Surg., 55: 735 (1968).

52. Mackler, B. F.: Role of soluble lymphocyte mediators in malignant tumor destruction. Lancet ii: 297 (1971).

53. Mathé, G. Amiel, J. L., Schwarzenberg L., Schneider, M., Cattan, A. Schlumberger, J. R., Hayat, M., and de Vassal, F.: Active immunotherapy for acute lymphoblastic leukemia. Lancet, i: 697 (1969)

54. McDevitt, H. O., and Benacerraf, B.: Genetic control of specific immune responses. Advan. Immunol., II: 31 (1969).

55. Meyer, J. J., Ribi, E. E., Azuma, I., and Zbar, B.: Biologically active components of mycobacterial cell walls. II. Suppression and repression of strain 2 guinea pig hepatoma. J. Natl. Cancer Inst., 52: 103 (1974).

56. Morton, D. L., Eilber, F. R., Malgren, R. A., and Wood, W. C.: Immunological factors which influecne response to immunotherapy in malignant melanoma. Surgery 68: 158, (1970).

57. Oettgen, H., Old, L., Farrow, J., Valentine, F., Lawrence, H. S., and Thomas, L. Effects of transfer factor in cancer patients. J. Clin. Invest., 50: 71 a (1971).

58. Old, L. J., Benacerraf, B., Clarke, D. A., Carswell, E. A., and Stockert, E.: The role of the reticuloendothelial system in the host reaction to neoplasia. Cancer Res., 21: 1281 (1961).

59. Oppenheim, J. J., Relationship of in vitro lymphocyte transformation to delayed hypersensitivity in guinea pigs and man. Fed. Proc., 27: 21 (1968).

60. Oren, M. E., and Herberman, R. B.: Delayed cutaneous hypersensitivity reactions to membrane extracts of human tumor cells. Clin. Exp. Immunol., 9 45 (1971).

61. Penn, I., and Starzl, T. E.: Malignant tumors arising de novo in immunosuppressed organ transplant recipients. Transplantation 14: 407 (1972).

62. Permain, G., Lycette, R. R., and Fitzgerald, P. H.: Tuberculin induced mitosis in peripheral blood leukocytes. Lancet, i: 637 (1963).

63. Pick, E., Krejci, K., Cech, K., and Turk, J. L.: Interaction between "sensitized lymphocytes" and antigen in vitro. $\mathrm{l}$. The release of a skin reactive factor. Immunology 17: 741 (1969).

64. Rosenberg, S. A., David, J. R., Palutke, M., and Rosenberg, J. C.: In vitro assay for detecting cellular immunity in man: Application to the study of human cancer immunity. Proc. Amer. Ass. Cancer Res., 12: 324 (1971).

65. Rule, A. H., Strauss, E., Vandevoorde, S., and Janowitz, H. D.: Tumorassociated (CEA-reacting) antigen in patients with inflammatory bowel disease. N. Engl. J. Med., 287: 24 (1972).

66. Scholossman, S. F.: The immune response: Some unifying concepts. N. Engl. J. Med., 277: 1355 (1967)

67. Sinkovics, J., Dreyer, D. A., Sherati, E., Cabiness, J. R., and Shullenberger, C. C.: Cytotoxic lymphocytes. I. Destruction of neoplastic cells by lymphocytes in cultures of human origin. Tex. Rep. Biol. Med., 29: 227 (1971).

68. Sjögren, H. O., Hellström, I., Bansal, S. C., and Hellström, K. E.: Suggestive evidence that the "blocking antibodies" of tumor-bearing individuals may be antigen-antibody complexes. Proc. Natl. Acad. Sci., U. S. A., 68: 1372 (1971) 
69. Smith, R. T. Tumor-specific immune mechanisms. N. Engl. J. Med., 278: 1207 $1268,1326(1968)$

70. Smith, R. T.: Possibilities and problems of immunologic intervention in cancer N. Engl. J. Med., 287: 439 (1972)

71. Soborg, $\mathbf{M}$. and Bendixen, G.: Human lymphocyte migration as a parameter of hypersensitivity. Acta, Med. Scand., 181: 247 (1967).

72. Solowey, A. C., and Rapaport, F. T.: Immunologic responses in cancer patients Surg. Gynecol. Obstet., 121: 756 (1965).

73. Sparks, F. C., Silverstein, M. J., Hunt, J. S., Haskell, C. M., Peck, Y. H., and Morton, D. L.: Complications of BCG immunotherapy in patients with cancer. N. Engl. J. Med. 289: 827 (1973)

74. Stewart, T. H. M.: The presence of delayed hypersensitivity reaction in patients toward cellular extracts of their malignant tumors. I. The role of tissue antigen nonspecific reactions of nuclear material, and bacterial antigen as a cause for this phenomenon. Cancer, 23: 1368 (1969).

75. Stjernswärd, J., Almgard, L. E., Franzen, S., von Schreeb, T., and Waldström, L. B.: Tumor destructive cellular immunity to renal carcinoma. Clin. Exp. Immunol., 6: 963 (1970)

76. Stjernswärd, J., and Clifford, P.: Tumor distinctive cellular immune reactions against autochthonous cancer. In: L. Severi: Immunity and Tolerance on Oncogenesis. Perugia, Div. Cancer Res., p. 749 (1970).

77. Stjernswärd, J., Clifford, P., and Singh, S., and Svedmyr, E.: Indications of cellular immunological reactions against autochthonous tumor in cancer patients studied in vitro. E. Afr. Med. J., 7: 484 (1968)

78. Stjernswärd, J.. Johansson, B., Svedmyr, E., and Sundblad, R.: Indication of tumor-specific cellbound immunological reactivity and depressed general reactivity in a pair of twins. Clin. Exp. Immunol., 6: 429 (1970).

79. Straus, E., Vernace, S., Janowtiz, H., and Paronetto, F.: Migration of peripheral leukocytes in the presence of carcinoembryonic antigen: Studies in patients with chronic inflammatory diseases of the intestines and carcinoma of the colon and pancreas. Proc. Soc. Exp. Biol. Med., 148: 494 (1975)

80. Thomas, L.: In: H. S. Lawrence: Cellular and Humoral-Aspects of Hypersensitivity States, p. 529 (H. Hoeber, New York, 1959).

81. Tripodi, D., Parks, L. C., and Brugmans, J.: Drug-induced restoration of cutaneous delayed hypersensitivity in anergic patients with cancer. N. Engl. J. Med., 289: 354 (1973).
82. Vanky, F. Stjernswärd, J., and Nilsonne, V.: Cellular immunity to human sarcoma. J. Natl. Cancer Inst. $46: 1145$ (1971)

83. Webster, D. J. T., and Hughes, L. E.: Levamisole. Lancet $i: 389$ (1975).

84. Wara, D. W., Goldstein, A. L., Doyle, N. E.. and Amman, A. J.: Thymosin activity in patients with cellular immunodeficiency. N. Engl. J. Med., 292: 70 (1975)

85. Ward, P. A., Remold, H. G., and David, J. R.: Leukotactic factor produced by sensitized lymphocyte. Science 163: 1079 (1969).

86. Ward, P. A., Remold, H. G., and David, J. R.: The production of antigen stimulated lymphocytes of a leukotactic factor distinct from migration inhibitor factor. Cell Immunol. $1: 162$ (1970).

87. Watkins, E. Jr. Ogata, Y., Anderson, L. L., Watkins, E., III, and Waters, M F.: Activation of host lymphocytes cultured with cancer cells heated with neuraminidase. Nature New Biol., 23l: 85 (1971).

88. Wheelock, E. F.: Interferon-like virus inhibitor induced in human leukocytes by phytohemagglutinin. Science, 149: 310 (1965).

89. Wolberg, W. H., and Goelzer, M. L.: In vitro assay of cell-mediated immunity in human cancer. Definition of leukocyte migration inhibitory factor. Nature, 229: 632 (1971).

90. Wybran, J., Levin, A. S., Spitler, L. E., and Fudenberg, H. H.: Rosette forming cells, immunologic deficiency disease and transfer factor. N. Engl. J Med., 288: $710(1973)$.

91. Zaldivar, N. M., Papageorgiou, P. S., Kaffe, S., and Glade, P. R.: The use of transfer factor in a patient with agammaglobulinemia. Pediat. Res. 9: 541 (1975).

92. This paper was part of the American Pediatric Society Symposium on Immunologic and Genetic Aspects of Cancer.

93. This work was supported in part by Research Grant IR01 AI12475 from the National Institutes of Allergy and Infectious Diseases; Contract no. NO1CB-33912 from the National Cancer Institute of the National Institutes of Health, United States Public Health Service; and by a grant from the Goldhirsch Foundation, New York.

94. Requests should be addressed to: P. R. Glade, M.D., Professor of Pediatrics, Division of Pediatric Infectious Disease and Immunology, University of Miami School of Medicine, P.O. Box 520875, Miami, Fla. 33152 (USA)

95. Accepted for publication October 31, 1975.

\title{
Electrolyte Abnormalities in Very Low Birthweight Infants
}

\author{
G. M. DAY, 1. C. RADDE, (32) J. W. BALFE, AND G. W. CHANCE \\ Research Institute, Divisions of Neonatology, Endocrinology, and Nephrology, The Hospital for Sick \\ Children; Department of Paediatrics, University of Toronto, Toronto, Ontario, Canada
}

Extract

In 30 very low birth weight (VLBW) infants, fed a commercial formula at $200 \mathrm{ml} / \mathrm{kg} / 24 \mathrm{hr}$ to provide $160 \mathrm{cal} / \mathrm{kg} / 24 \mathrm{hr}$, hyponatremia (plasma $\mathrm{Na}^{+}<130 \mathrm{mEq}$ /liter) occurred in 23 patients ( 14 appropriate for gestational age (AGA), 9 small for gestational age (SGA)) between the ages of 2 and 6 weeks. In five infants the hyponatremia recurred even after adequate correction of the deficit. Calcium supplementation given to 14 of the infants did not affect the incidence or severity of hyponatremia. In A GA infants, the hyponatremia was more severe than in SGA babies.

Hyperkalemia (plasma $\mathrm{K}^{+}>5.5 \mathrm{mEq} /$ liter) was more common in AGA than in SGA infants (16/17 AGA, 7/13 SGA). Decrease in mean plasma chloride concentrations was proportionate to the decrease in mean $\mathrm{Na}^{+}$.

Urinary $\mathrm{Na}^{+}$averaged $1.0 \mathrm{mEq} / \mathrm{kg} / 24 \mathrm{hr}$ and was equal between groups during the first balance at a mean age of 18 days. In subsequent balances it appeared to decrease more rapidly in noncalcium-supplemented than in calcium-supplemented infants, but the difference was not significant $(P<0.1)$. Fecal excretion of $\mathrm{Na}^{+}$ did not differ between groups.

Symptoms were unrelated to the degree of hyponatremia or hyperkalemia and were nonspecific.

Hyponatremia in A GA infants occurred in $50 \%$ of instances when growth was $\leq 0.75 \mathrm{~cm} /$ week, whereas it occurred in only $13.5 \%$ of infants when growth exceeded $1 \mathrm{~cm} /$ week $(P<0.01)$. A similar trend in SGA infants was not statistically significant $(P<0.2)$.

\section{Speculation}

The hyponatremia in VLBW infants between weeks 2 and 6 of postnatal age is due to a combination of high urinary losses relative to plasma levels, insufficient intake due to the relatively low $\mathrm{Na}^{+}$ content of certain infant formulas and coprecipitation of $\mathrm{Na}^{+}$in 